\title{
Bakterielle Genregulation
}

\section{Freund oder Feind? - Die zwei Ge- sichter von Photorhabdus luminescens}

\section{NAZZARENO DOMINELLI, RALF HEERMANN \\ INSTITUT FÜR MOLEKULARE PHYSIOLOGIE, MIKROBIOLOGIE UND WEINFORSCHUNG, UNIVERSITÄT MAINZ}

Photorhabdus luminescens is an enteric bacterium with two faces: on the one hand these bacteria live in symbiosis with soil nematodes, on the other hand they are highly pathogenic for insects. The population is also phenotypically heterogeneous: one part lives in symbiosis with nematodes, the other part symbiotically interacts with plants. Cell-cell communication, inter-kingdom signaling, and other gene regulation processes are behind the complex decision of being friend or foe.

DOI: $10.1007 / \mathrm{s} 12268-021-1662-9$

(C) Die Autoren 2021
Es passiert in der dunklen Erde, im Wald, in Ackerböden, im Sand der Küste und in unseren Vorgärten: Winzige Fadenwürmer (Nematoden) mit dem Namen Heterorhabditis bacteriophora suchen nach Opfern. Um erfolgreich zu töten, tragen sie eine gefährliche Waffe in ihrem Vorderdarm: insektenpathogene Bakterien der Art Photorhabdus luminescens. So sind die Nematoden bestens gerüstet, um ahnungslose Insektenlarven zu

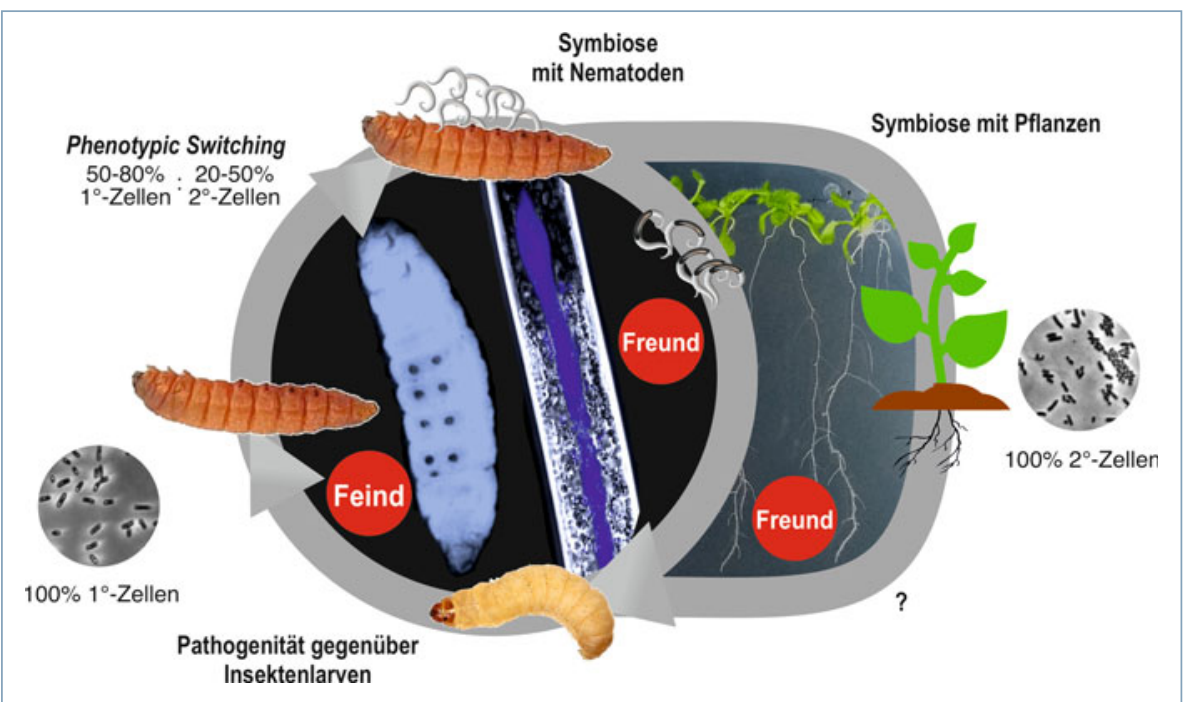

$\Delta$ Abb. 1: Die Lebenszyklen von Photorhabdus luminescens. $1^{\circ}$ - und $2^{\circ}$-Zellen sind hochpathogen gegenüber Insektenlarven. Ausschließlich die $1^{\circ}$-Zellen leben in Symbiose mit den Nematoden (Freund). Letztere infizieren Insektenlarven mit $1^{\circ}$-Zellen und töten sie durch die bakteriell produzierten Toxine (Feind) effizient und schnell. Nachdem der Kadaver des Opfers durch die Bakterien verstoffwechselt ist, wechseln 20-50 Prozent der $1^{\circ}$-Zellen zum $2^{\circ}$-Phänotyp. Während die restlichen $1^{\circ}$-Zellen wieder von den Nematoden aufgenommen werden (Freund), interagieren $2^{\circ}$-Zellen symbiotisch mit Pflanzenwurzeln und verteidigen diese vor dem Befall durch phytopathogene Pilze (Freund). Ob die $2^{\circ}$-Zellen wieder zu $1^{\circ}$-Zellen konvertieren können, ist bisher unklar. erlegen. Ist das Opfer ausgemacht, geht alles blitzschnell: Die Nematoden bohren ein Loch in die Haut des Insekts und schlüpfen ins Innere. Dort angekommen, würgen sie die Bakterien hinaus und lassen sie ihre Arbeit verrichten.

$P$. luminescens ist ein echter Killer, ausgestattet mit einem großen Arsenal verschiedener Toxine und Sekundärmetabolite, die das Opfer innerhalb kurzer Zeit töten. In der Folge bilden die Bakterien das Enzym Luziferase, sodass das tote Insekt luminesziert - der „leuchtende Tod“ hat zugeschlagen.

Danach produzieren die Bakterien diverse Exoenzyme, die den Kadaver des Opfers zu einer nährstoffreichen „Suppe“ zersetzen, die nun sowohl die Bakterien als auch die Nematoden nutzen können. Die Bakterien unterstützen zudem ihre Symbiosepartner in Fortpflanzung und Entwicklung durch die Produktion noch unbekannter Sekundärmetabolite. Durch die Produktion von Antibiotika und Fungiziden verteidigt Photorhabdus sein neues Habitat vor dem Befall durch andere Bodenmikroorganismen. Sind die Nährstoffe aufgebraucht, nehmen die Nematoden die Bakterien wieder auf, verlassen das aufgezehrte Insekt und bringen ihre Biowaffe in ein neues Opfer (Abb. 1).

Freund oder Feind - bakterielle phänotypische Heterogenität

Doch $P$. luminescens ist außerhalb der Nematoden nicht allen Eukaryoten gegenüber feindlich gestimmt. So profitieren Pflanzen von einer Interaktion mit den Bakterien [1]. Die Gram-negativen Enterobakterien kommen in zwei verschiedenen phänotypischen Formen vor, den primären $\left(1^{\circ}\right)$ und sekundären $\left(2^{\circ}\right)$ Zellen [2]. Genetisch sind beide Zellformen identisch, unterscheiden sich aber in verschiedenen phänotypischen Eigenschaften. Nur die $1^{\circ}$-Zellen produzieren Antibiotika, Pigmente und sind biolumineszent. Einer der wichtigsten Unterschiede der beiden Zellformen ist jedoch, dass ausschließlich $1^{\circ}$-Zellen mit den Nematoden eine Verbindung eingehen können. Während des Infektionszyklus vollziehen einzelne 
$1^{\circ}$-Zellen einen Phasenwechsel, sodass nach etwa 28 Tagen fast die Hälfte der Population zum $2^{\circ}$-Phänotyp gewechselt ist. Die $2^{\circ}$-Zellen verbleiben im Boden, wenn die neue Generation der Nematoden den aufgezehrten Insektenkadaver verlassen hat. Die $2^{\circ}$-Zellen sind beweglicher als $1^{\circ}$-Zellen und werden von Pflanzenwurzelexsudaten angelockt [3]. Sie gehen eine spezifische Interaktion mit den Wurzeln ein und produzieren hier eine noch unbekannte Substanz, die die Pflanzen vor dem Befall phytopathogener Pilze schützt [1]. Weiterhin bewirkt die Kolonisierung der Bakterien eine Verzweigung des Wurzelwachstums, was für die Pflanze wachstumsfördernd ist [1]. Da die $2^{\circ}$-Zellen ebenfalls insektenpathogen sind, könnten sie die Pflanzenwurzeln auch vor Fraß durch Insektenlarven schützen. Die Rückkehr vom $2^{\circ}$ zum $1^{\circ}$-Phänotyp ist wahrscheinlich, aber bisher noch nie beobachtet worden.

\section{Bakterielle Kommunikation}

Um zwischen Freund und Feind zu unterscheiden, müssen Bakterien kommunizieren. Sie können sowohl mit ihren Artgenossen als auch mit anderen Bakterienarten oder ihren eukaryotischen Wirten über kleine Moleküle Informationen austauschen. In Gram-negativen Bakterien erfolgt diese Kommunikation oft über LuxR-Rezeptoren [4]. P. luminescens besitzt 40 verschiedene solcher LuxR-Rezeptoren, mehr als jede andere bisher entdeckte Bakterienart [5]. Untereinander kommunizieren $P$. luminescens- ${ }^{\circ}$-Zellen mit $\alpha$-Pyronen, auch Photopyrone (PPY) genannt, die von der Photopyronsynthase PpyS synthetisiert werden. Der LuxR-Rezeptor PluR sensiert diese und steuert somit die Verklumpung der Zellen und damit die Virulenz (Abb. 2, [6]). Wie $2^{\circ}$-Zellen untereinander kommunizieren, ist unklar. Die nahverwandte Art $P$. asymbiotica, die beim Menschen Hautinfektionen verursacht, nutzt keine PPY zur Kommunikation, sondern Dialkyresorzinole (DAR) [7]. Die Änderung der Kommunikationsweise von PPY zu DAR wird als entscheidender evolutiver Schritt von Insektenzu Humanpathogenität gesehen.

Insgesamt 35 der LuxR-Rezeptoren in P. luminescens besitzen eine PAS4-Signalbindedomäne, die sie wahrscheinlich für die Kommunikation mit eukaryotischen Wirten nutzen [5]. Erste Ergebnisse zeigen, dass die Bakterien über einen dieser PAS4-LuxRRezeptoren Signalmoleküle aus Wachsmottenlarven wahrnehmen (unveröffentlichte Daten). Andere der PAS4-LuxR-Solos könn-

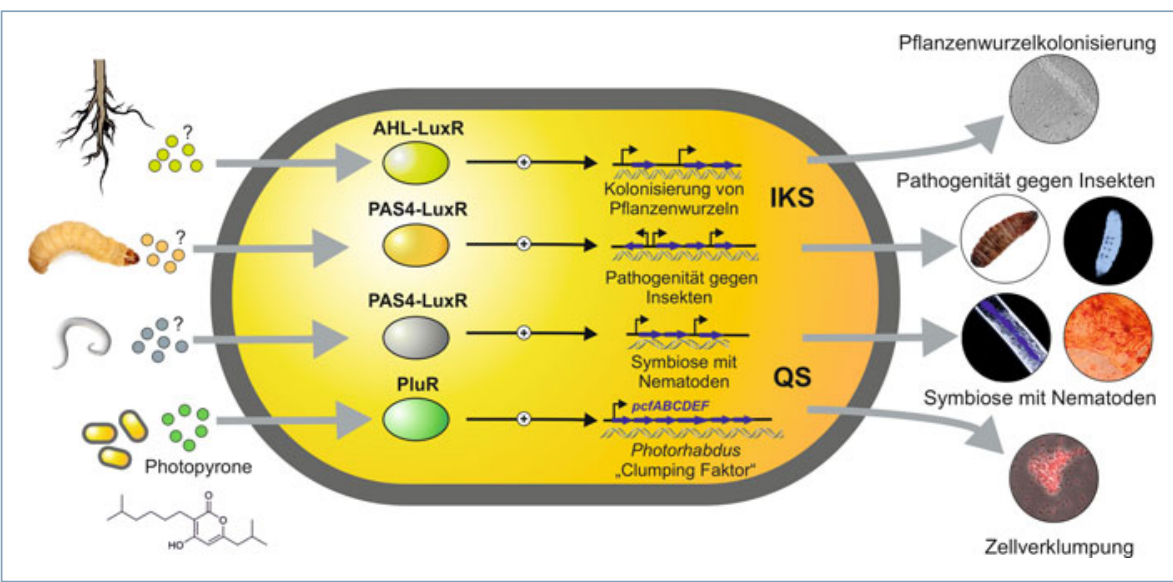

A Abb. 2: Bakterielle Kommunikation. Über eine Vielfalt von LuxR-Rezeptoren mit unterschiedlichen Signalbindedomänen (PluR, PAS4, AHL) können Photorhabdus luminescens-Zellen sowohl untereinander als auch mit ihren eukaryotischen Wirten kommunizieren. Dabei wird der LuxRRezeptor PluR für die Kommunikation der Bakterien untereinander genutzt, während die PAS4LuxR-Rezeptoren wahrscheinlich beim inter-kingdom signaling mit den eukaryotischen Wirten (Insekten und Nematoden) eine Rolle spielen. Dem AHL-LuxR-Rezeptor SdiA wird eine Funktion bei der Kommunikation mit den Pflanzen zugeschrieben. Die chemische Natur des zugehörigen Signalmoleküls ist ausschließlich für PluR bekannt. QS: Quorum sensing; IKS: inter-kingdom signaling.

ten sie auch für die Erkennung der Nematoden nutzen (Abb. 2). P. luminescens besitzt außerdem einen LuxR-Solo, der eine AcylHomoserinlakton(AHL)-Bindedomäne besitzt und homolog zu SdiA ist [5]. Da einige pflanzenpathogene Bakterien über SdiA pflanzliche Signalmoleküle und keine AHL wahrnehmen, könnte bei P. luminescens SdiA ebenfalls für die Kommunikation zwischen Pflanze und $2^{\circ}$-Zellen wichtig sein (Abb. 2). Insgesamt deutet die hohe Anzahl der LuxRRezeptoren in P. luminescens darauf hin, dass die Bakterien echte Kommunikationstalente sind und viele „Sprachen“ nutzen, um Freund und Feind zu unterscheiden.

\section{Regulation von Freundschaft und Feindschaft}

Die Entscheidung für das phenotypic switching von $1^{\circ} \mathrm{zu} 2^{\circ}$ muss gut in der Population abgestimmt sein. Einer der Hauptregulatoren des switching ist der LysR-ähnliche Regulator HexA (Abb. 3), ein Repressor für $1^{\circ}$-spezifische Gene [8]. So ist die Konzentration von HexA in $2^{\circ}$-Zellen im Vergleich zu $1^{\circ}$-Zellen erhöht. HexA reguliert die Expression einiger Zielgene direkt und anderer indirekt über kleine regulatorische RNAs [8]. Die Expression von hexA selbst unterliegt der Kontrolle des RNA-Chaperons Hfq, sodass die Steuerung der HexA-Kopienzahl posttranskriptional reguliert wird [9]. Die zeitliche Koordination des switching-Prozesses wird über das Sensorkinase/Antwortregulatorsystem AstS/AstR reguliert (Abb. 3, [3]). Wel- chen Reiz die Sensorkinase wahrnimmt, um den Antwortregulator AstR zu phosphorylieren, ist unklar. Da das universelle Stressprotein UspA unter Kontrolle von AstS/AstR steht, wird vermutet, dass globaler Stress zum Einleiten des switching führt [3].

Die Pigmentierung in $1^{\circ}$-Zellen wird durch Anthraquinone (AQ) hervorgerufen, welche die Kolonien der Bakterien sowie auch die toten Insektenlarven rot erscheinen lassen [10]. Der AQ-Biosyntheseweg wird vom antABCDEFGHI-Operon codiert, dessen Expression unter Kontrolle des Regulators AntJ steht (Abb. 3). Die Kopienzahl von AntJ ist in einzelnen $1^{\circ}$ Zellen erhöht und damit heterogen verteilt [11]. Weiterhin spielen bei der Aktivierung des phenotypic switching zwei Regulatoren der XRE-Familie eine besondere Rolle - XreR1 und XreR2 (Abb. 3, [12]). Die Kopienzahl von XreR1 ist in $1^{\circ}{ }^{\circ}$, die von XreR2 in $2^{\circ}$-Zellen erhöht. Das Regulatorpaar scheint durch positive Autoregulation einen epigenetischen switch zu konstituieren, indem XreR1 die Expression von xreR2 reprimiert und XreR2 die Expression seines eigenen Gens durch direkte Wechselwirkung mit XreR1 steigert [12].

Wir sind erst am Anfang zu verstehen, wie komplex die Regulation der phänotypischen Heterogenität in P. luminescens ist. Es ist unklar, welche Signale zum Auslösen des switching führen und was letztendlich die heterogene Aktivierung einzelner regulatorischer Gene bewirkt. 


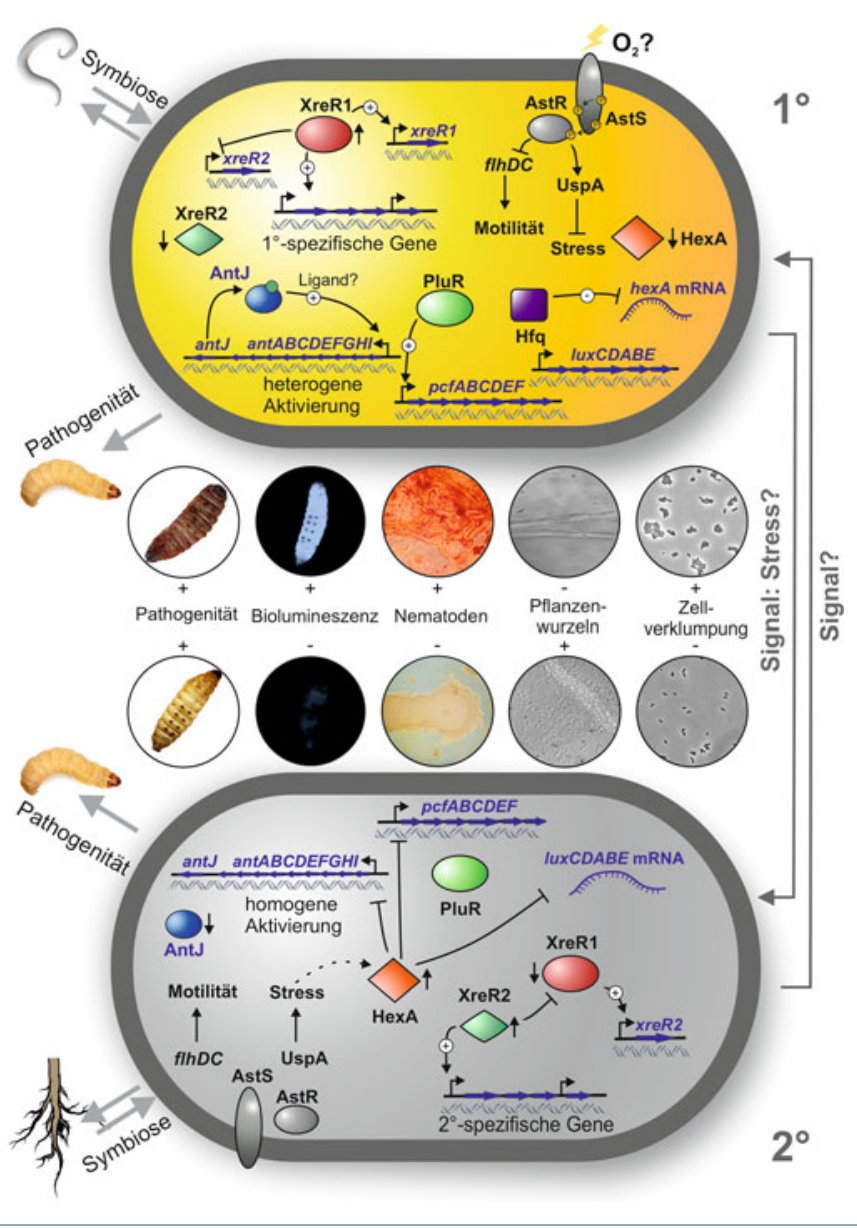

\section{Vielfältige Anwendung in der Biotechnologie}

$P$. luminescens ist nicht nur ein exzellenter Modellorganismus, um bakterielle Kommunikation, Pathogenität oder phänotypische Heterogenität zu studieren. Die Bakterien sind auch vielseitig biotechnologisch nutzbar. So werden die entomopathogenen Nematoden bereits als Bioinsektizide für die Bekämpfung von Schädlingen eingesetzt. Weiterhin sind die Bakterien Reservoir für eine Vielzahl neuer Naturstoffe, die als Wirkstoffe in Biotechnologie und Medizin zum Einsatz kommen könnten. $2^{\circ}$-Zellen könnten zudem zukünftig als Biostimulanzien zur Ertragssteigerung in der Agrarbiotechnologie dienen.

$P$. luminescens - Freund oder Feind? Eine Frage, die ganz klar vom Blickwinkel des Betrachters abhängt. Aus Sicht der Insekten sind die Bakterien sicherlich ein todbringender Feind, aber aus Sicht der Nematoden und der Pflanzen eher ein Freund. Und für uns Menschen? Aufgrund ihrer vielseitigen biotechnologischen Anwendungsmöglichkeiten ist $P$. luminescens für uns sicherlich ein sehr guter Freund.

\section{Danksagung}

Die Arbeiten wurden durch die DFG gefördert (SPP1617, HE5247/5-2; HE5247/4-2).

$\triangle$ Abb. 3: Regulation phänotypischer Heterogenität in Photorhabdus luminescens. Das phenotypic switching von $1^{\circ} \mathrm{zu} 2^{\circ}$ und die daraus resultierenden Phänotypen (siehe Bildmitte) werden durch ein komplexes Zusammenspiel unterschiedlicher Regulatoren kontrolliert. Dabei spielt der Regulator HexA eine zentrale Rolle. Dieser ist in $2^{\circ}$-Zellen in hohen Konzentrationen vorhanden und inaktiviert $1^{\circ}$-spezifische Gene, die wichtig für Pigmentierung, Biolumineszenz, Zellverklumpung und die Nematoden-Interaktion sind. Außerdem spielen zwei XRE-Regulatoren eine wichtige Rolle beim Wechsel von $1^{\circ} \mathrm{zu} 2^{\circ}$. Während XreR 1 in $1^{\circ}$-Zellen in hohen Konzentrationen vorhanden ist und $1^{\circ}$-spezifische Gene aktiviert, wird dieser in $2^{\circ}$-Zellen durch direkte Wechselwirkung mit XreR2 reprimiert. Dabei werden $2^{\circ}$-spezifische Gene in $2^{\circ}$-Zellen aktiviert, die für die Kolonisierung von Pflanzenwurzeln essenziell sind. Das Sensorkinase/ Antwortregulatorsystem AstS/AstR kontrolliert dabei das zeitliche Einleiten des switching-Prozesses. Das globale Signal für den Phasenwechsel von einzelnen Zellen in einer Population sowie die Signale für die einzelnen am Prozess beteiligten Regulatoren sind nicht bekannt.

\section{Literatur}

[1] Regaiolo A, Dominelli N, Andresen $\mathrm{K}$, Heermann R (2020) The biocontrol agent and insect pathogen Photorhabdus luminescens interacts with plant roots. App Env Microbiol 86: 234215

[2] Eckstein S, Heermann R (2019) Regulation of phenotypic switching and heterogeneity in Photorhabdus luminescens cell populations. J Mol Biol 431: 45594568

[3] Eckstein S, Dominelli N, Brachmann A, Heermann R (2019) Phenotypic heterogeneity of the insect pathogen Photorhabdus luminescens: insights into the fate of sec-

ondary cells. App Env Microbiol 85: e01910-19 [4] Tobias NJ, Brehm J, Kresovic D et al. (2020) New vocabulary for bacterial communication. ChemBioChem 21: 759768

[5] Brameyer S, Kresovic D, Bode HB, Heermann R (2014) LuxR solos in Photorhabdus species. Front Cell Infect Microbiol 4: 166

[6] Brachmann AO, Brameyer S, Kresovic D et al. (2013) Pyrones as bacterial signaling molecules. Nat Chem Biol 9: 573-578

[7] Brameyer S, Kresovic D, Bode HB, Heermann R (2015) Dialkylresorcinols as bacterial signaling molecules. Proc Natl Acad Sci USA $112: 572-577$

[8] Langer A, Moldovan A, Harmath C et al. (2017) HexA is a versatile regulator involved in the control of phenotypic heterogeneity of Photorhabdus luminescens. PLoS ONE 12: $\mathrm{e} 0176535$

[9] Neubacher N, Tobias NJ, Huber M et al. (2020) Symbiosis, virulence and natural-product biosynthesis in entomopatho- genic bacteria are regulated by a small RNA. Nature Microbiology 5: 1481-1489

[10] Brachmann AO, Joyce SA, Jenke-Kodama H et al. (2007) A type II polyketide synthase is responsible for anthraquinone biosynthesis in Photorhabdus luminescens. ChemBioChem 8: 1721-1728

[11] Heinrich AK, Glaeser A, Tobias NJ et al. (2016) Heterogeneous regulation of bacterial natural product biosynthesis via a novel transcription factor. Heliyon 2: e00197 [12] Eckstein S, Brehm J, Seidel M et al. (2021) Two novel XRE-like transcriptional regulators control phenotypic heterogeneity in Photorhabdus luminescens cell populations. BMC Microbiol 21: 63

Funding note: Open Access funding enabled and organized by Projekt DEAL. Open Access: Dieser Artikel wird unter der Creative Commons Namensnennu 4.0 International Lizenz veröffentlicht, welche die Nutzung, Vervielfältigung, Bearbeitung, Verbreitung und Wiedergabe in jeglichem Medium und For ellaubl, sofern Sie den/die urspranglichen Autor(en) und die Quele Fon ordnungsgen angeben, ob Anderungen vorgenommen wurden. Die in diesem Artikel genannten Creative Commons Lizenz, sofern sich aus der Abbildungslegen nichts anderes ergibt. Sofern das betreffende Material nicht unter der genannten Creative Commons Lizenz steht und die betreffende Handlung nicht nach gesetzlichen Vorschriften erlaubt ist, ist für die oben aufgeführten Weiterverwendungen des Materials die Einwilligung des jeweiligen Weiterverwendungen des Materials die Einwilligung des jeweiligen
Rechteinhabers einzuholen. Weitere Details zur Lizenz entnehmen Sie bitte der

\section{Korrespondenzadresse:}

Prof. Dr. Ralf Heermann

Johannes Gutenberg-Universität Mainz Institut für Molekulare Physiologie Mikrobiologie und Weinforschung

Biozentrum II

Hanns-Dieter-Hüsch-Weg 17

D-55128 Mainz

heermann@uni-mainz.de

Ralf Heermann
Studium der Biologie und Promotion im Fach Mikrobiologie. 2001-2004 Postdoc, Uni-
versität Osnabrück und TU Darmstadt. 2004-2010 Wissenschaftlicher Assistent und
Habilitation im Fach Mikrobiologie der LMU München. 2010-2018 Akademischer
(Ober-)Rat und Arbeitsgruppenleiter an der LMU. Seit 2018 Universitätsprofessor für
Mikrobiologie an der Universität Mainz und seit 2021 Assoziierter Professor am Insti-
tut für Biotechnologie und Wirkstoff-Forschung (IBWF) in Mainz.

